\title{
The effect of cyclic hygrothermal conditions on the stresses near the surface of a thick composite pipe
}

\author{
Frédéric Jacquemin, Alain Vautrin* \\ Mechanical and Materials Engineering Department, Ecole des Mines de Saint-Etienne, 158, cours Fauriel, F-42023 Saint-Etienne cedex 02, France
}

\begin{abstract}
It is necessary to estimate the moisture concentration and the hygrothermal internal stress fields to evaluate the durability of thick composite pipes submitted to cyclic environmental conditions. After some time, the moisture concentration, induced by temperature and relative-humidity cycles, is permanent within the pipe and periodic close to the inner and outer surfaces. The hygrothermal stresses induced are computed by using the classical equations of solid mechanics and assuming a hygrothermoelastic orthotropic behaviour for every ply. The aim of this paper is to model the effects of the periodic boundary conditions on the hygrothermal stresses.
\end{abstract}

Keywords: A. Polymer-matrix composites; B. Hygrothermal effect; Durability

\section{Introduction}

We consider a thick laminated pipe, whose outer and inner radii are $a$ and $b$, respectively, submitted to temperature and relative humidity cycles of the same period, $\tau$. Assuming that the thermal equilibrium is reached instantaneously, the temperature is considered to be uniform over the thickness of the pipe at any time.

The moisture concentration, $c(r, t)$, is solution of the following system with Fick's Eq. (1) and boundary and initial conditions Eq. (2):

$\frac{\partial c}{\partial t}=D(t)\left[\frac{\partial^{2} c}{\partial r^{2}}+\frac{1}{r} \frac{\partial c}{\partial r}\right], a<r<b$

$\left\{\begin{array}{l}c(a, t)=c_{\mathrm{a}}(t) \text { and } c(b, t)=c_{\mathrm{b}}(t) \\ c(r, 0)=0\end{array}\right.$

The diffusion coefficient $D(t)$ is only a time dependent function which depends on the temperature through an Arrhénius' law. $c_{\mathrm{a}}(t)$ and $c_{\mathrm{b}}(t)$ are the cyclic boundary concentrations related to the relative humidity. $D(t), c_{\mathrm{a}}(t)$ and $c_{\mathrm{b}}(t)$ are periodic time functions of the same period $\tau$.

The general solution of this problem, studied by Jacquemin and Vautrin [1], comprises a transient part,

\footnotetext{
* Corresponding author.

E-mail address: vautrin@emse.fr (A. Vautrin).
}

which converges towards a permanent solution (3) within the pipe and a fluctuating part which converges towards a periodic solution of period $\tau$ in the vicinity of the external surfaces.

$c(r)=\frac{\int_{0}^{\tau} D(t) c_{\mathrm{b}}(t) d t}{\int_{0}^{\tau} D(t) \mathrm{d} t}+\frac{\int_{0}^{\tau} D(t)\left(c_{\mathrm{a}}(t)-c_{\mathrm{b}}(t)\right) d t}{\ln \frac{a}{b} \int_{0}^{\tau} D(t) d t} \ln \left(\frac{r}{b}\right)$

Therefore, the permanent solution (3) is only valid up to a distance from the edge where the cyclic boundary conditions are applied. The extent $e_{0}$ of the periodic solution, which will be determined by using a finite difference scheme, has been estimated by Verchery [2] for a semi-infinite plate, depends on the diffusion coefficient, the temperature and the period of the cycles:

$e_{0}=2 \sqrt{\pi \int_{0}^{\tau} D(t) \mathrm{d} t}$

\section{Mechanical problem}

In this part, the hygrothermal stresses are computed by using the classical equations of solid mechanics for every ply at any time: constitutive laws of hygrothermoelastic orthotropic materials (5), strain/displace- 
ment relationship, compatibility and equilibrium equations.

$\sigma=\mathbf{L}:\left(\varepsilon-\alpha\left(T-T_{0}\right)-\beta\left(m-m_{0}\right)\right)$

with $m=\frac{m_{\text {water }}}{m_{\mathrm{s}}}=\frac{c}{\rho_{\mathrm{s}}}$

$c$ and $\rho_{\mathrm{s}}$ are, respectively, the moisture concentration and the mass density of the dry material.

Introducing $L_{0}$, we consider the following reduced variables:

$\bar{\sigma}=\sigma / L_{0}, \mathbf{L}=L / L_{0},(\bar{w}, \bar{u}, \bar{v})=(w, u, v) / b, \bar{r}=r / b$

Displacement with respect to $x$ and $\theta$, respectively $\bar{u}(\bar{x}, \bar{r})$ and $\bar{v}(\bar{x}, \bar{r})$ are then expressed:

$$
\left\{\begin{array}{c}
\bar{u}(\bar{x}, \overline{\mathrm{r}})=-\overline{\mathrm{S}}_{\mathrm{r} \theta \mathrm{xr}} \frac{R_{3}}{\bar{r}}+\bar{S}_{\mathrm{xrxr}} R_{4} \ln \bar{r}+R_{1} \bar{x}+R_{5}, \\
\bar{v}(\bar{x}, \overline{\mathrm{r}})=\mathrm{R}_{2} \bar{x} \bar{r}-\frac{\bar{S}_{\mathrm{r} \theta \mathrm{r} \theta}}{2} \frac{R_{3}}{\bar{r}}-\bar{S}_{\mathrm{r} \theta \mathrm{xr}} R_{4}+R_{6} \bar{r}, \\
R_{1}, R_{2}, R_{3}, R_{4}, R_{5}, \mathrm{R}_{6} \text { are constants and } \mathrm{S}=\mathbf{L}^{-1} .
\end{array}\right.
$$

The radial component of the displacement field, $\bar{w}$, satisfies the following expression:

$$
\begin{aligned}
& \bar{r}^{2} \frac{\partial^{2} \bar{w}}{\partial \bar{r}^{2}}+\bar{r} \frac{\partial \bar{w}}{\partial \bar{r}}-\frac{\bar{L}_{\theta \theta}}{\bar{L}_{\mathrm{rr}}} \bar{w}= \\
& \frac{\bar{r}\left[\left(\bar{L}_{\mathrm{x} \theta}-\bar{L}_{\mathrm{xr}}\right) R_{1}+\left(\bar{L}_{\mathrm{s} \theta}-2 \bar{L}_{\mathrm{rs}}\right) R_{2} \bar{r}\right]}{\bar{L}_{\mathrm{rr}}}+ \\
& \frac{\bar{r}\left[\left(I_{1}-I_{2}\right)\left(T-T_{0}\right)+\left(K_{1}-K_{2}\right)\left(m-m_{0}\right)+K_{1} \bar{r} \frac{\partial m}{\partial \bar{r}}\right]}{\bar{L}_{\mathrm{rr}}}
\end{aligned}
$$

Table 1

Hygroscopic properties

\begin{tabular}{ll}
\hline Diffusion coefficient $\left(\mathrm{mm}^{2} / \mathrm{s}\right)$ & $D(t)=0.57 \exp (-4993 / T(t))$ \\
\hline $\begin{array}{l}\text { Ambient moisture } \\
\text { concentration }\left(\mathrm{kg} / \mathrm{m}^{3}\right)\end{array}$ & $c=0.2385 \mathrm{H}$ \\
\hline
\end{tabular}

with, $I_{1}=\bar{L}_{\mathrm{xr}} \alpha_{\mathrm{xx}}+\bar{L}_{\mathrm{r} \theta} \alpha_{\theta \theta}+\bar{L}_{\mathrm{rr}} \alpha_{\mathrm{rr}}+\bar{L}_{\mathrm{rs}} \alpha_{\mathrm{x} \theta}, I_{2}=\bar{L}_{\mathrm{x} \theta} \alpha_{\mathrm{xx}}$ $+\bar{L}_{\theta \theta} \alpha_{\theta \theta}+\bar{L}_{\mathrm{r} \theta} \alpha_{\mathrm{rr}}+\bar{L}_{\mathrm{s} \theta} \alpha_{x \theta}, \quad K_{1}=\bar{L}_{\mathrm{xr}} \beta_{\mathrm{xx}}+\bar{L}_{\mathrm{r} \theta} \beta_{\theta \theta}+$ $\bar{L}_{\mathrm{rr}} \beta_{\mathrm{rr}}+\bar{L}_{\mathrm{rs}} \beta_{\mathrm{x} \theta}, K_{2}=\bar{L}_{\mathrm{x} \theta} \beta_{\mathrm{xx}}+\bar{L}_{\theta \theta} \beta_{\theta \theta}+\bar{L}_{\mathrm{r} \theta} \beta_{\mathrm{rr}}+\bar{L}_{\mathrm{s} \theta} \beta_{\mathrm{x} \theta}$.

\subsection{Radial component of the displacement field within the pipe}

The general solution of the differential Eq. (7) is the sum of a solution of the homogeneous equation and of a particular solution. Considering the permanent moisture concentration (3), we obtain the radial component of the displacement field within the pipe:

$$
\begin{aligned}
& \bar{w}=R_{7} \bar{r} \sqrt{\frac{\bar{L}_{\theta \theta}}{\bar{L}_{\mathrm{rr}}}}+R_{8} \bar{r}^{-\sqrt{\frac{\bar{\varphi}_{\theta \theta}}{\bar{L}_{\mathrm{rr}}}}}+ \\
& \frac{\left(\bar{L}_{\mathrm{x} \theta}-\bar{L}_{\mathrm{xr}}\right) R_{1} \bar{r}}{\bar{L}_{\mathrm{rr}}\left(1-\frac{\bar{L}_{\theta \theta}}{\bar{L}_{\mathrm{rr}}}\right)}+\frac{\left(\bar{L}_{\mathrm{s} \theta}-2 \bar{L}_{\mathrm{rr}}\right) R_{2} \bar{r}^{2}}{\bar{L}_{\mathrm{rr}}\left(4-\frac{\bar{L}_{\theta \theta}}{\bar{L}_{\mathrm{rr}}}\right)}+ \\
& \frac{\left(I_{1}-I_{2}\right)\left(T-T_{0}\right) \bar{r}}{\bar{L}_{\mathrm{rr}}\left(1-\frac{\bar{L}_{\theta \theta}}{\bar{L}_{\mathrm{rr}}}\right)}-\frac{\left(K_{1}-K_{2}\right) m_{0} \bar{r}}{\bar{L}_{\mathrm{rr}}\left(1-\frac{\bar{L}_{\theta \theta}}{\bar{L}_{\mathrm{rr}}}\right)}+ \\
& \frac{\left(K_{1}-K_{2}\right) \bar{r}}{\rho_{\mathrm{s}} \bar{L}_{\mathrm{rr}}\left(1-\frac{\bar{L}_{\theta \theta}}{\bar{L}_{\mathrm{rr}}}\right)} \frac{\int_{0}^{\tau} D(t) c_{\mathrm{b}}(t) \mathrm{d} t}{\int_{0}^{\tau} D(t) \mathrm{d} t} \\
& {\left[\frac{\left(K_{1}-K_{2}\right) \bar{r} \ln \bar{r}+K_{1} \bar{r}}{\rho_{\mathrm{s}} \bar{L}_{\mathrm{rr}}\left(1-\frac{\bar{L}_{\theta \theta}}{\bar{L}_{\mathrm{rr}}}\right)}-\frac{2\left(K_{1}-K_{2}\right) \bar{r}}{\rho_{\mathrm{s}} \bar{L}_{\mathrm{rr}}\left(1-\frac{\bar{L}_{\theta \theta}}{\bar{L}_{\mathrm{rr}}}\right)^{2}}\right]} \\
& \frac{\int_{0}^{\tau} D(t)\left(c_{\mathrm{a}}(t)-c_{\mathrm{b}}(t)\right) \mathrm{d} t}{\ln \frac{a}{b} \int_{0}^{\tau} D(t) \mathrm{d} t} \\
& \text { for } \frac{\bar{L}_{\theta \theta}}{\bar{L}_{\mathrm{rr}}} \neq 1, \frac{\bar{L}_{\theta \theta}}{\bar{L}_{r r}} \neq 4 \text {. }
\end{aligned}
$$

\subsection{Radial component of the displacement field in the vicinity of the surfaces}

In the vicinity of the surfaces, the periodic concentration is determined by using a finite difference scheme. To propose a close form solution of the displacement, we subdivide the extent of the periodic concentration and assume on each subdivision a parabolic moisture concentration (9):

Table 2

Mechanical properties in the orthotropic reference frame $(1,2,3)$

\begin{tabular}{llllllllll}
\hline Material & $E_{1}(\mathrm{Gpa})$ & $E_{2}, E_{3}(\mathrm{Gpa})$ & $\nu_{12}, \nu_{13}$ & $\nu_{23}$ & $G_{12}(\mathrm{Gpa})$ & $\alpha_{1}\left(\mathrm{~K}^{-1}\right)$ & $\alpha_{2}, \alpha_{3}\left(\mathrm{~K}^{-1}\right)$ & $\beta_{1}$ & $\beta_{2}, \beta_{3}$ \\
\hline T300/5208 & 181 & 10.3 & 0.28 & 0.43 & 7.17 & $0.0210^{-6}$ & 22.5 & $10^{-6}$ & 0 \\
\hline
\end{tabular}


$c_{\mathrm{i}}=A_{\mathrm{i}}^{\prime} \bar{r}^{2}+B_{\mathrm{i}}^{\prime} \bar{r}+C_{\mathrm{i}}^{\prime}$

Thus, we obtain the radial component of the displacement field, solution of Eq. (7), for each subdivision:

$$
\begin{aligned}
& \bar{w}=R_{7} \bar{r} \sqrt{\frac{\bar{L}_{\theta \theta}}{\bar{L}_{\mathrm{rr}}}}+R_{8} \bar{r}^{-} \sqrt{\frac{\bar{L}_{\theta \theta}}{\overline{\mathrm{r}}_{\mathrm{rr}}}}+\frac{\left(\bar{L}_{\mathrm{x} \theta}-\bar{L}_{\mathrm{xr}}\right) R_{1} \bar{r}}{\bar{L}_{\mathrm{rr}}\left(1-\frac{\bar{L}_{\theta \theta}}{\bar{L}_{r r}}\right)} \\
& +\frac{\left(\bar{L}_{\mathrm{s} \theta}-2 \bar{L}_{\mathrm{rs}}\right) R_{2} \bar{r}^{2}}{\bar{L}_{\mathrm{rr}}\left(4-\frac{\bar{L}_{\theta \theta}}{\bar{L}_{\mathrm{rr}}}\right)}+\frac{\left(I_{1}-I_{2}\right)\left(T-T_{0}\right) \bar{r}}{\bar{L}_{\mathrm{rr}}\left(1-\frac{\bar{L}_{\theta \theta}}{\bar{L}_{r r}}\right)} \\
& -\frac{\left(K_{1}-K_{2}\right) m_{0} \bar{r}}{\bar{L}_{\mathrm{rr}}\left(1-\frac{\bar{L}_{\theta \theta}}{\bar{L}_{\mathrm{rr}}}\right)}+\frac{K_{1}}{\rho_{\mathrm{s}} \bar{L}_{\mathrm{rr}}}\left[\frac{B_{i}^{\prime} \bar{r}^{2}}{\left(4-\frac{\bar{L}_{\theta \theta}}{\bar{L}_{\mathrm{rr}}}\right)}+\frac{2 A_{\mathrm{r}}^{\prime} \bar{r}^{3}}{\left(9-\frac{\bar{L}_{\theta \theta}}{\bar{L}_{\mathrm{rr}}}\right)}\right] \\
& +\frac{\left(K_{1}-K_{2}\right)}{\rho_{\mathrm{s}} \bar{L}_{\mathrm{rr}}}\left[\frac{C_{\mathrm{i}}^{\prime} \bar{r}}{\left(1-\frac{\bar{L}_{\theta \theta}^{\prime}}{\bar{L}_{\mathrm{rr}}}\right)}+\frac{B_{\mathrm{r}}^{2}}{\left(4-\frac{\bar{L}_{\theta \theta}^{\prime}}{\bar{L}_{\mathrm{rr}}}\right)}+\frac{\left(9-\frac{\bar{L}_{\theta \theta}}{\bar{L}_{\mathrm{rr}}}\right)}{(4)}\right.
\end{aligned}
$$

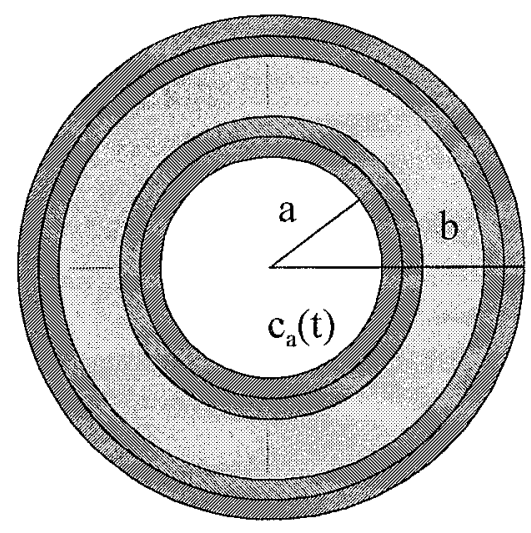

$\theta$

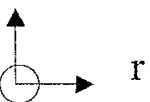

$\mathrm{x}$

$\mathrm{c}_{\mathrm{b}}(\mathrm{t})$

Fig. 1. Hollow laminated cylinder.

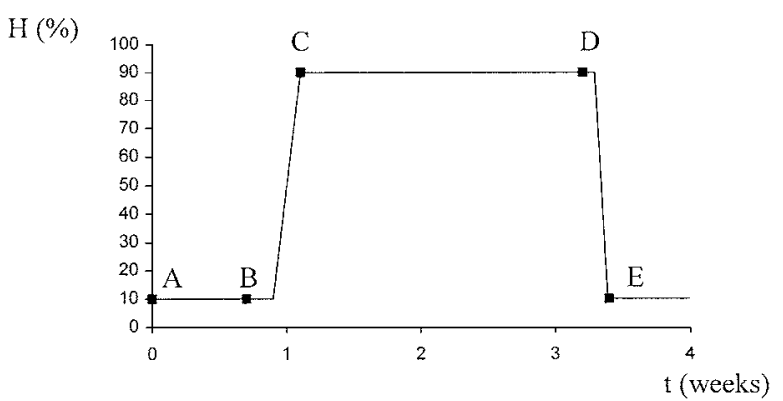

Fig. 2. Cyclic concentration on the surfaces.
Finally, the displacement through every ply depends on eight constants to be determined : $R_{i}$ for $i=1 . .8$.

\subsection{Determination of eight constants per ply}

The eight constants are determined from the following conditions:

- rigid body motions restrained;

- continuity of the displacement components at each interply;

- continuity of the transverse shear stress at each interply;

- continuity of the normal stress at each interply and its nullity on the boundaries surfaces;

- global force balance of the pipe.

\section{Case study}

We consider a thick pipe made up of five carbon/ epoxy plies of equal thickness alternatively oriented at $+55^{\circ}$ or $-55^{\circ}$ versus the longitudinal axis. The outer and inner radii are, respectively, $a=10 \mathrm{~mm}$ and $b=30$ $\mathrm{mm}$. The hygroscopic properties [3] and the mechanical properties [4] are presented in Tables 1 and 2.The pipe is homogeneous from the hygroscopic point of view, every ply has the same hygroscopic properties (Table 1), but it is heterogeneous from the mechanical point of view because of the different orientations of the plies. The pipe is submitted to relative humidity (Fig. 1 and Fig. 2) and temperature (Fig. 3) cycles of 4 week period.

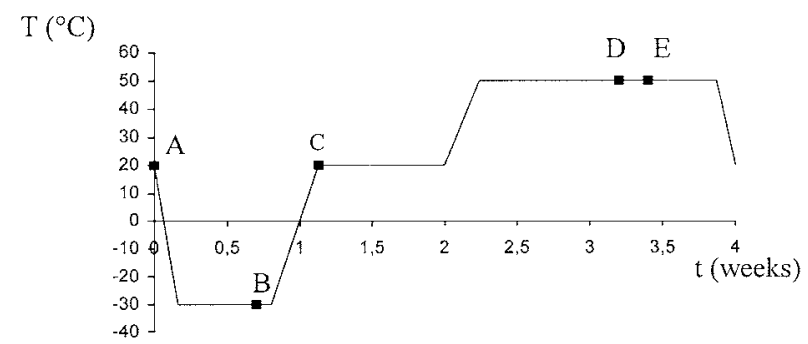

Fig. 3. Temperature cycle.

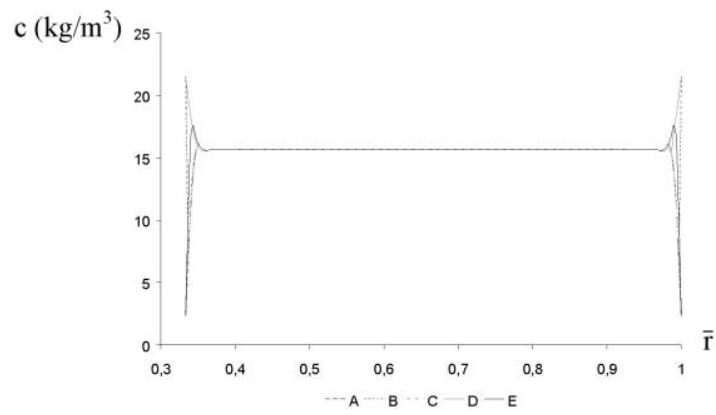

Fig. 4. Moisture concentration for different points of the cycles. 


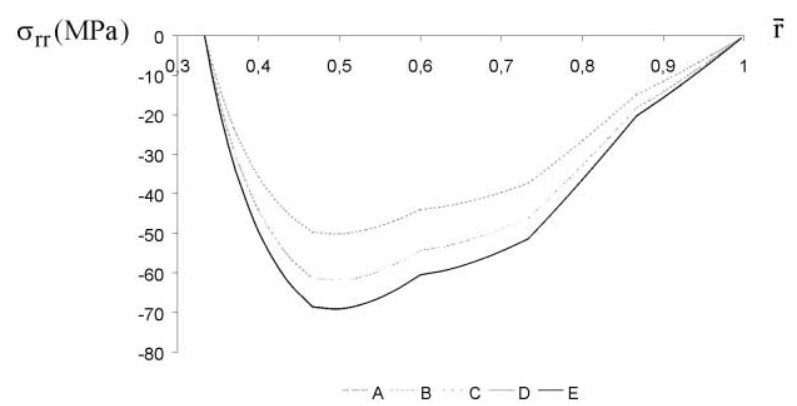

Fig. 5. Radial stress for different points of the cycles.

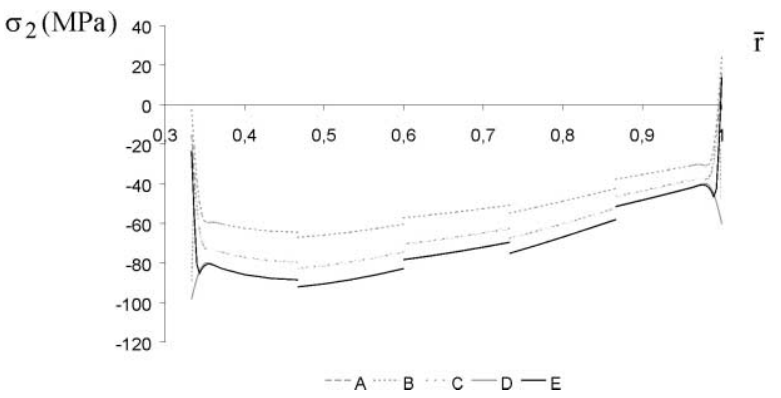

Fig. 6. Normal in-plane stress in the transverse direction to the fibres.

Fig. 4 shows that the oscillations of the periodic concentration disappear at a distance $e_{0}$ from the edge. Therefore, at a distance $e_{0}$ from the edge the permanent concentration holds with a constant value because of the symmetrical hygrothermal loading. We observe that fluctuating concentration gradients are important for the points $C$ and $E$ where the relative humidity changes roughly. Figs. 5 and 6 depict the radial stress and the normal ply stress in the transverse direction to the fibres, respectively. We observe that the periodic concentration gradients have not any influence on the radial stress but induce strong gradients of the normal ply stress. In this periodic concentration regions, the normal ply stress gradients are so important that theirs values firstly negatives become positives. The radial stress and the normal ply stress within the pipe are dependent on the temperature changing: for $(A, C)$ and $(D, E)$, corresponding to identical temperature but to different relative humidity, the stresses are identical. The temperature decreases between $A$ and $B$ induce tensile stresses and the temperature increase between $C$ and $D$ induce compressive stresses.

\section{Conclusion}

We propose an approach which allows to measure the influence of the periodic field, close to the surfaces, of the moisture concentration on the internal stresses for thick laminated pipes. For the internal stresses induced by cyclic hygrothermal conditions, we dissociate the thermal effects and the hygroscopic effects. This solution provides a helpful tool for the design of thick composite pipes under hygrothermal fatigue, since it leads to the knowledge of the stress evolution which can be strong within a narrow region near to the surfaces where holds the periodic moisture concentration.

\section{References}

[1] Jacquemin F, Vautrin A. Thick laminated pipes submitted to cyclic environmental conditions. In: 9th European Conference on Composite Materials, Brighton 2000.

[2] Verchery G. Moisture diffusion in polymer matrix composites with cyclic environmental conditions. In: 5th European Conference on Composite Materials, Bordeaux 1992.

[3] Loos AC, Springer GS. Moisture absorption of graphite-epoxy composition immersed in liquids and in humid air. In: Springer GS, editor. Environmental effects on composite materials, Springer G. S., Technomic 1981, p. 51-62.

[4] Tsai SW. Composite design., Think composites, 1987. 